\title{
An Inequality for the Volume of Inscribed Ellipsoids
}

\author{
L. G. Khachiyan \\ Computing Center of the USSR Academy of Sciences, \\ Vavilov Street 40, Moscow 117367, USSR
}

\begin{abstract}
Let $K$ be a convex body in $R^{n}$, and let $x^{*} \in$ int $K$ be the center of the ellipsoid of the maximal volume inscribed in the body. An arbitrary hyperplane through $x^{*}$ cuts $K$ into two convex bodies $K^{+}$and $K^{-}$. We show that $w\left(K^{ \pm}\right) / w(K) \leq$ $0.844 \ldots$, where $w(\cdot)$ is the volume of the inscribed ellipsoid.
\end{abstract}

\section{Introduction}

Let $K$ be a convex body in $R^{n}$. It is known [1] that among all the ellipsoids $E$ contained in the body, $E \subseteq K$, there exists a unique ellipsoid $E^{*}=E^{*}(K)$ of the maximal volume. We call $E^{*}$ the ellipsoid inscribed in the body and denote by

$$
w(K)=\max \{\text { vol } E \mid E \text { is an ellipsoid, } E \subseteq K\}
$$

the volume of $E^{*}$ viewed as a function of $K$. The center $x^{*}=x^{*}(K)$ of the inscribed ellipsoid is called the center of the body.

Let $x^{*}$ be the center of a convex body $K$. Since $x^{*} \in$ int $K$, an arbitrary hyperplane $P=\left\{x \in R^{n} \mid p^{t}\left(x-x^{*}\right)=0\right\}$ through $x^{*}$ splits $K$ into two convex bodies

$$
K^{+}=\left\{x \in K \mid p^{*}\left(x-x^{*}\right) \geq 0\right\}, \quad K^{-}=\left\{x \in K \mid p^{\prime}\left(x-x^{*}\right) \leq 0\right\}
$$

We show that the size $w(\cdot)$ of the splinters $K^{+}, K^{-}$is always at least $\alpha=0.844 \ldots$ times smaller than the size of the initial body, i.e., for all $n, K$, and $P$

$$
\max \left\{\frac{w\left(K^{+}\right)}{w(K)}, \frac{w\left(K^{-}\right)}{w(K)}\right\} \leq \alpha=0.844 \ldots
$$


Remark. In [2] inequality (1) was proven with a worse constant of shrinkage, $\alpha=0.888$... It may be conjected that the best possible value of $\alpha$ in inequality (1) equals $0.5 e^{1 / 2}=0.824 \ldots$. The latter value is attained as $n \rightarrow \infty$ in case $K$ is a spherical cone with $P$ parallel to the base.

Remark. If $K$ is a polyhedra defined by a finite system of linear inequalities, then $w(K)$ can be determined in polynomial time with an arbitrary fixed absolute accuracy [2]. The same computational problem for determining $\operatorname{vol}(K)$ is \# $\mathrm{P}$ hard.

Remark. Replacing the "lower ellipsoidal volume" $w(K)$ by the "upper ellipsoidal volume" $W(K)=\min \{\operatorname{vol} E \mid E$ is an ellipsoid, $K \subseteq E\}$, and taking an $n$-dimensional Euclidean ball as $K$, we get

$$
\max \left\{\frac{W\left(K^{+}\right)}{W(K)}, \frac{W\left(K^{-}\right)}{W(K)}\right\} \geq 1-\frac{1}{2 n}
$$

under an arbitrary choice of $x^{*}$ and $P$. Thus, for $W(\cdot)$ inequality (1) can hold for all $n$ only trivially, i.e., with $\alpha=1$.

\section{Proof of Inequality (1)}

An arbitrary ellipsoid $E$ in $R^{n}$ can be given by the pair $(a, A)$, where $a \in R^{n}$ is the center of the ellipsoid and $A$ is an $n \times n$ symmetric positive definite matrix: $E=\left\{x \in R^{n} \mid(x-a)^{t} A^{-2}(x-a) \leq 1\right\}$. This ellipsoid is the image of the Euclidean unit ball $\|y\|=\left(y^{t} y\right)^{1 / 2} \leq 1$ under the transformation $A$, shifted to the point $a$, i.e.,

$$
E=\left\{x \in R^{n} \mid x=a+A y,\|y\| \leq 1\right\} .
$$

In particular, the support function and the volume of the ellipsoid $E \sim(a, A)$ are given by the expressions

$$
\begin{aligned}
& g_{E}(c)=\max \left\{c^{t} x \mid x \in E\right\}=c^{t} a+\left\|c^{t} A\right\|, \\
& \text { vol } E=v(n) \operatorname{det} A,
\end{aligned}
$$

where $v(n)$ stands for the volume of the $n$-dimensional Euclidean unit ball. First we need the following

Lemma 1. Let $E^{*} \sim\left(a^{*}, A^{*}\right)$ be the ellipsoid inscribed in a convex body $K$ and let $E \sim(a, A)$ be an arbitrary ellipsoid contained in the body. Then

$$
\operatorname{vol} E / \text { vol } E^{*} \leq x \cdot \exp (1-x)
$$


for any $x$ in the interval

$$
\min _{c \in R^{n}} \frac{\left\|c^{2} A\right\|}{\left\|c^{i} A^{*}\right\|}=\lambda \leq x \leq \Lambda=\max _{c \in R^{n}} \frac{\left\|c^{z} A\right\|}{\left\|c^{t} A^{*}\right\|}
$$

Proof. Since the contents of the lemma is invariant under affine transformations, we may assume that $E^{*}$ is the unit ball $A^{*}=I=\operatorname{diag}(1, \ldots, 1)$ and $A=$ $\operatorname{diag}\left(\lambda_{1}, \ldots, \lambda_{n}\right)>0$, where $\lambda_{1}, \ldots, \lambda_{n}$ are the semiaxes of $E$. Then $\lambda=\min \lambda_{i}$ and $\Lambda=\max \lambda_{i}, i \in\{1, \ldots, n\}$. Since the ellipsoids $E^{*}$ and $E$ are contained in $K$, it follows that for any $t \in[0,1]$ the ellipsoid $E(t) \sim(1-t)\left(a^{*}, A^{*}\right)+t(a, A)$ is contained in $K$ as well. Consider the function

$$
f(t)=\ln (\operatorname{vol} E(t) / \operatorname{vol} E(0))=\sum_{i \in\{1, \ldots, n\}} \ln \left(1+t\left(\lambda_{i}-1\right)\right)
$$

The maximality of the volume of $E^{*}=E(0)$ implies $f^{\prime}(0) \leq 0$, i.e.,

$$
\operatorname{tr} A=\sum_{i \in\{1, \ldots, n\}} \lambda_{i} \leq n
$$

Therefore

$$
\operatorname{vol} E / \text { vol } E^{*}=\prod_{i \in\{1, \ldots, n\}} \lambda_{i} \leq \prod_{i \in\{1, \ldots, n\}} \lambda_{i} \cdot \exp \left(1-\lambda_{i}\right)
$$

Since $x \exp (1-x) \leq 1$ for all $x$ and the minimum of the function $x \cdot \exp (1-x)$ over $[\lambda, \Lambda]$ is attained in the endpoints of the interval, the proof is completed.

We now want to prove inequality (1). Since $K^{ \pm} \rightarrow K^{\mp}$ as $p \rightarrow-p$, it suffices to prove that $w\left(K^{-}\right) / w(K) \leq \alpha=0.844 \ldots$. Let $E^{*}$ and $E^{-}$be the ellipsoids inscribed in $K$ and $K^{-}$. Inequality (1) is invariant under affine transformations and we may assume without loss of generality that $E^{*} \sim(a, D)$ and $E \sim\left(-a, D^{-1}\right)$, where $D=\operatorname{diag}\left(d_{1}, \ldots, d_{n}\right)>0$. Since the centers of $E^{*}$ and $E^{-}$are placed in the points $a$ and $-a$, it follows that $0 \in$ int $K$. In this case the body $K$ can be defined by the system of linear inequalities $K=\left\{x \in R^{n} \mid c^{t} x \leq 1, c \in K^{0}\right\}$, where $K^{0}$ is the polar set of covectors. The ellipsoids $E^{*}$ and $E^{-}$are contained in $K$ if and only if

$$
\begin{aligned}
& g_{E^{*}}(c)=c^{t} a+\left\|c^{t} D\right\| \leq 1, \quad \forall c \in K^{0}, \\
& g_{E}(c)=-c^{t} a+\left\|c^{t} D^{-1}\right\| \leq 1, \quad \forall c \in K^{0} .
\end{aligned}
$$

Multiplying the last inequalities for a fixed $c \in K^{0}$ we get

$$
c^{t} c \leq\left\|c^{t} D\right\|\left\|c^{t} D^{-1}\right\| \leq\left(1+c^{t} a\right)\left(1-c^{t} a\right)=1-\left(c^{t} a\right)^{2},
$$

i.e.,

$$
c^{t} c+\left(c^{t} a\right)^{2}=\left\|c^{t} \sqrt{I+a a^{t}}\right\|=g_{E}(c) \leq 1, \quad \forall c \in K^{0}
$$


where $E$ is the ellipsoid given by the pair $\left(0, \sqrt{I+a a^{7}}\right)$. Hence the ellipsoid $E \sim\left(0, \sqrt{I+a a^{t}}\right)$ is contained in $K$. Note that vol $E=v(n) \cdot \sqrt{1+a^{t} a}$. We now wish to apply Lemma 1 to the ellipsoids $E^{*}$ and $E$. To this end, let us show that

$$
\min _{c \in R^{n}} \frac{\| c^{t} \sqrt{I+a a^{t} \|}}{\left\|c^{t} D\right\|}=\lambda \leq \frac{\sqrt{1+a^{t} a}}{d} \leq \Lambda=\max _{c \in R^{n}} \frac{\left\|c^{t} \sqrt{I+a a^{t}}\right\|}{\left\|c^{t} D\right\|},
$$

where $d=\max \left(d_{1}, \ldots, d_{n}\right)$. Indeed, let $d=d_{1}$. Setting $c_{1}=(1,0, \ldots, 0)$ we have

$$
\lambda \leq \frac{\| c_{1}^{t} \sqrt{I+a a^{t} \|}}{\left\|c_{1}^{t} D\right\|}=\frac{\sqrt{1+a_{1}^{2}}}{d} \leq \frac{\sqrt{1+a^{2} a}}{d} .
$$

On the other hand, the choice $c_{2}=a /\|a\|$ yields

$$
\frac{\sqrt{1+a^{t} a}}{d} \leq \frac{\sqrt{1+a^{t} a}}{\left\|c_{2}^{t} D\right\|}=\frac{\left\|c_{2}^{t} \sqrt{I+a a^{t}}\right\|}{\left\|c_{2}^{t} D\right\|} \leq \Lambda
$$

and we can apply Lemma 1:

$$
\frac{\operatorname{vol} E}{\operatorname{vol} E^{*}}=\frac{\sqrt{1+a^{t} a}}{d_{1} \cdots d_{n}} \leq \frac{\sqrt{1+a^{t} a}}{d} \cdot \exp \left(1-\frac{\sqrt{1+a^{t} a}}{d}\right)
$$

To complete the proof the last observation is needed: since the center $a$ of the ellipsoid $E^{*}$ lies on the boundary of $K^{-}$, it follows that

$$
a \notin \text { int } E^{-}=\left\{x \in R^{n} \mid x=-a=D^{-1} y,\|y\|<1\right\} .
$$

In other words, $2\|a D\| \geq 1$, and consequently $4 d^{2}\left(a^{\prime} a\right) \geq 1$. Therefore

$$
\begin{aligned}
\frac{w\left(K^{-}\right)}{w(K)} & =\frac{\operatorname{vol} E^{-}}{\operatorname{vol} E^{*}}=\frac{1}{\left(d_{1} \cdots d_{n}\right)^{2}} \leq \frac{1}{d^{2}} \cdot \exp \left(2-\frac{2 \sqrt{1+a^{t} a}}{d}\right) \\
& \leq \frac{1}{d^{2}} \cdot \exp \left(2-\frac{2 \sqrt{1+1 / 4 d^{2}}}{d}\right) \leq \max _{d^{2}}\left\{\frac{1}{d^{2}} \cdot \exp \left(2-\frac{\sqrt{4 d^{2}}+1}{d^{2}}\right)\right\} \\
& =0.844 \ldots
\end{aligned}
$$

the last maximum being attained in the root $d^{2}=1.517 \ldots$ of the cubic equation $4 d^{6}-3 d^{4}-4 d^{2}-1=0$.

\section{References}

1. L. Danzer, D. Laugwitz, H. Lenz, Uber das Löwnersche Ellipsoid und sein Analogen unter den einem Eikorper einbeschriebener Ellipsoiden, Arch. Math. 8 (1957).

2. S. P. Tarasov, L. G. Khachiyan, I. I. Erlich, The method of inscribed ellipsoids, Soviet Math. Dokl. 37(1) (1988).

Received June 1, 1988. 